\title{
<Title>Chapter 15: Do Creative Skills Future-Proof Your Job? Creativity and the Future of Work in an Age of Exponential Technological Advancement ${ }^{1}$ Ruth Bridgstock ${ }^{2}$, Russell Tytler $^{3}$ and Peta White ${ }^{4}$
}

\section{$<$ Heading 1 $>$ Introduction}

It is the year 2030. Jacob has just embarked on his third career change in 15 years, and is enrolled in a postgraduate course with the aim of becoming a nostalgist, which is one of a 'new wave' of creative job roles that came about with massive advances to digital technologies.

People now live longer and there is a strong demand in virtual reality experiences that allow the elderly to relive the best parts of their lives. Nostalgists combine interior design, digital research and virtual-reality development to create these experiences (Tytler et al., 2019). While Jacob is studying, he is also working part-time as a sub-contracted aged-care worker, for which he was awarded a foundational qualification last year. Prior to this, Jacob worked as a plasterer, but it was becoming too hard to compete with automated plastering services, and so he decided to change.

Jacob's story was created from themes in research interviews conducted in 2019 with experts in science, technology and social trends. While specifics such as job titles may not stand the test of time, the presence of consistent themes in the interviews helps to clarify how work and careers might unfold in the future, and which capabilities are likely to be important. This chapter shares the findings of those interviews.

The speed, extent and impact of technological changes on the world of work has been subjected to much debate during the last few years. Popular media, government and consultancy reports, and academic literature alike abound with deterministic predictions of 'robots coming for our jobs', arguing that between $20 \%$ and $60 \%$ of job roles will disappear over coming decades under the influence of automation, robotics and artificial intelligence (AI). Others emphasise the creation of entirely new jobs that are based on technological advances. A third category of future-focused literature emphasises qualitative and task-based changes to job roles. As machines become able to perform some tasks better and more cost-effectively than humans, human jobs are predicted to become either more repetitive and low-skilled, or more complex and high-skilled. Work arrangements are already changing, with movement from a 'traditional'

\footnotetext{
${ }^{1}$ This chapter includes excerpts from Tytler, R., Bridgstock, R. S., White, P., Mather, D., McCandless, T., \& Grant-Iramu, M. (2019). The 100 Jobs of the Future Report available at www. 100jobsof the future.com. The funding of Deakin Unversity, Griffith University and Ford are gratefully acknowledged.
}

${ }^{2}$ ORCID iD: 0000-0003-0072-2815

Centre for Learning Futures, Griffith University

r.bridgstock@griffith.edu.au

${ }^{3}$ ORCID iD: 0000-0003-0161-7240

School of Education, Deakin University

${ }^{4}$ ORCID iD: 0000-0002-0225-5934

School of Education, Deakin University 
model of full-time, continuing employment activity at a place of work to employment arrangements that are more flexible in a number of ways.

Creativity is one category of capability that has been suggested to be uniquely human, or at least less 'susceptible' to automation than other categories. In turn, job roles that involve significant creativity may also be less susceptible to automation. According to some sources, and in line with contemporary arguments about the creative economy (Bakhshi, Frey, \& Osborne, 2015; Cunningham \& Potts, 2015), such job roles may continue to add greater value in the future world of work than other roles. This chapter considers the proposition that creative skills and tasks 'future-proof' human work roles - or put another way, that creative workers possess distinctive and valuable creative capabilities that are unlikely to be replaced by machines. The discussion includes the likelihood that machines can become, or are becoming, creative in different ways and contexts, as well as exploring the ways that humans are creative, and how creativity adds value to our economy and society, now and into the future.

This chapter commences by exploring the contrasting predictions about technological advances and the future of work, along with the economic and social mediating forces that are thought to be influential. Then, it discusses different kinds of creativity and other 'uniquely human' capabilities in terms of the types of value that they add, and draws upon the study ' 100 Jobs of the Future' to characterise the potential importance and value of creativity and other skills in a highly technology-dominated world of work. Finally, the chapter asks some larger questions about the complementary roles that creative humans and machines might be able to play in meaningful future activities, such as critical and creative reasoning and design, which promote economic and environmental sustainability, and strengthen individual and community health and well-being (Gershenfeld, Gershenfeld, \& Cutcher-Gershenfeld, 2017).

\section{$<$ Heading 1>Technological Advances and the Changing Future (and Present) of Work}

Scholars argue that we are now experiencing the fourth industrial revolution (Schwab, 2016), also known as the second machine age (Brynjolfsson \& McAfee, 2014) and the third digital revolution (Gershenfeld et al., 2017). Such scholars suggest that we stand on the brink of an era in which AI, automation, robots and digital networks will transform the way we live, work and learn. Changing work roles and activities due to technological progress is not a new phenomenon. The Luddites of the first industrial revolution, afraid for their jobs, destroyed weaving machinery. In the 1970s and 1980s, thousands of typesetting compositors (workers who arranged movable type for printing presses) were made obsolete by computerisation. The difference in the early 21 st century seems to be that a diverse range of technological advancements are occurring both simultaneously and at an exponential rate, creating much larger, and more widespread, changes to work than ever before.

\section{$<$ Heading 2>Disappearing Jobs}

Over the last decade, discussions of work and employment have become dominated by predictions about technology-driven disappearance or creation of jobs, changes to tasks inside jobs, and change in the organisation and experiences of future work. Frey and Osborne's (2013) working paper set the tone for many of the initial predictive studies, suggesting that, based on the characteristics of existing roles and the predicted capabilities of computers over coming years, nearly half of the jobs in the United States (US) were at high risk of being automated in the next 20 years. This study was followed by several others using similar methodologies demonstrating that the technical potential for automation of job roles in the next few years is quite high. For instance, in Australia, in the next decade or so, around $40 \%$ of jobs are forecast to be strongly 
affected by computerisation and automation (Durrant-Whyte, McCalman, O'Callaghan, Reid, \& Steinberg, 2015; Edmonds \& Bradley, 2015). Looking at current work tasks rather than roles, Manyika et al. (2017) indicated that about half of work activities could be automated by 2030 .

\section{$<$ Heading 2>Rearranging Jobs}

Literature also suggests that digital technologies will also continue to change how work is arranged and experienced by workers. Retail, service and entertainment functions have already started to shift online or be offered through distributed networks. The gig economy relies on digital technologies to mediate between companies and workers who engage in freelance and casual work, and the ironically named 'sharing economy' enables commercial transactions between workers and customers, through platforms such as Airtasker, Uber and AirBnB. Crowdsourcing work platforms such as Amazon's MTurk distribute labour-intensive work to many unrelated workers.

These new work arrangements have been praised for their potential to enhance work-life balance and to offer flexibility to workers in terms of supporting them to set their own hours and conditions. However, such arrangements have also been strongly criticised for compromising job security and not offering leave and other benefits, thus, shifting many of the risks associated with employment onto the worker (World Economic Forum, 2018). Even in more 'conventional' employment situations where organisations employ workers directly, there may be a shift towards outsourcing of work, short and flexible contracts, and freelancing - all directly or indirectly enabled by digital technologies.

\section{$<$ Heading 2>Emerging and Newly Appearing Jobs}

While many frame the future of work pessimistically, others have pointed out that technology can enhance work lives, eliminating dangerous or boring work, and creating new job roles (c.f. Lent, 2018). Citing the example of the iPhone, Huws (2014) discusses how new technologies can create and support innumerable work roles right across the value chain and across many countries, from raw materials through to design, manufacture, sale and disposal. The same kinds of online distributed networks that are rearranging jobs are diversifying options for consumers and businesses to access goods and services, offering new avenues for commodification, and more jobs. Autor (2015) discusses the complementarities between automation and human labour that can actually increase productivity, earnings, and demand for workers.

In a striking counter-balance to the 'robots coming for our jobs' narrative, a sub-set of the work futures literature is now devoted to how new technologies have the potential to create new and exciting job roles that have not yet been thought of (e.g., Borland \& Coelli, 2017). This literature cites examples of job roles and entire fields where this has already occurred in the last few years, such as visual effects (VFX) compositors ${ }^{5}$ (in contrast to the printing press compositors already mentioned), other VFX artists, social media marketers and cybersecurity professionals. Tytler et al. (2019) recently published a study titled ' 100 Jobs for the Future', which aimed to inform educational curriculum development by interrogating technological and research advances, along with social and economic trends, to predict and describe potential job roles for the next 30 years. The study then analysed the key capability requirements for the

\footnotetext{
${ }^{5}$ Good VFX compositors are very much in demand in the 21 st century world of work. These are the VFX artists who integrate live-action and digital elements to create the final completed shot in a film or game.
} 
predicted work roles. Tytler et al.'s (2019) study will be discussed in more detail later in this chapter, in relation to creativity and creative capabilities for future work.

\section{$<$ Heading 2>Influences Beyond Technical Potential: Jobs Disappearing, Appearing and Rearranging}

There is a significant lack of accord among scholars about the impact of technological change on the future of work in the 21 st century. In part, this discord is because of the very complex influences that social and economic forces have on work, and also on technology development and application. For instance, Frey and Osborne (2013) moderated their initial estimate of a nearly 50\% role disappearance by documenting a range of forces that could slow or increase the pace of disruption of jobs by technology, such as the regulation and policy, economic conditions and public opposition. Similarly, Spencer (2018) considers the 'politics of production' that shape how technology operates in the economy, emphasising the role of ownership and the current political economy of capitalism on how technological advances and the future of work evolve. For instance, to the extent that people might be displaced by machines and, thus, be unemployed or underemployed with little or no income, they will also consume less. Beyond technology per se, such factors are potentially capable of modifying the primary drivers of large-scale social and economic change.

Mishel and Bivens (2017) discuss the importance of economic and social influences on work rather than an inexorable future "robot apocalypse" (p. 3). Specifically, the authors analyse the current state of income polarisation in the US and show that, to date, automation has actually had very little to do with this. Mishel and Bivens (2017) argue that rapid technological advances historically have been associated with some displacement of workers, but better outcomes for them overall. The authors do not discount the probability of technologically induced shifts in work and jobs in the future, but indicate that rapid technological advances need to be complemented by labour policies to improve equity and labour standards for workers.

Other discussions of the power of social and economic influences on the future of technology and work explore the rise in offshoring, migration and global employment practices, along with exploitation of tax opportunities and exposure (Ford, 2015; Neufeind, O'Reilly \& Ranft, 2018). Such trends could lead to a smaller and more precarious workforce, though at the same time labour shortages, for example through the retirement of baby boomers could compensate (e.g. Lent, 2018).

\section{$<$ Heading 2>All of the Above (and Maybe More)? A Complex Picture of the Future of Work}

Scholars tend to argue strongly for one position or another, but both 'optimistic' and 'pessimistic' views of technological advances and the future of work could well come to pass (e.g. Lent, 2018), possibly concurrently, while continuing to be mediated strongly by social and economic influences. Routine tasks are vulnerable to automation - and, indeed, some of these jobs (such as straightforward customer service and manufacturing roles) are already in decline (Autor, Levy, \& Murnane, 2003; Frey \& Osborne, 2013). However, some authors (Spencer, 2018; Spreitzer, Cameron, \& Garrett, 2017) propose that some jobs in the future, at least for a while, may be even more routine and repetitive. These are job roles that are very straightforward for humans, but are beyond the capabilities of machines, or for which it may be cheaper and quicker to employ a human being. For instance, some of the tasks that MTurk employs humans to do, often for a few cents each, are to categorise data, caption pictures, tag metadata, analyse sentiments and place ads in videos. Many of these tasks then feed machine-learning algorithms 
and teach AI systems. Workers who engage in these tasks, often through crowdsourcing platforms, are predicted to make up a large proportion of the human 'precariat' in the future (Standing, 2011).

While some scholars have assumed that today's white-collar or knowledge workers are likely to occupy highly valued roles in the future, studies have shown that these workers are not immune to automation. Susskind and Susskind (2016) explore the future of the professions, and demonstrate that a significant part of many of these roles can be automated. As Hughes (2017) points out, nurses with computers may provide cheaper diagnoses than doctors. On the other hand, there may also be created some more 'fulfilling jobs' for humans that require a wider range of more complex skills to either work with, complement or circumvent technology. The nurse who trains to work with computer diagnosis may be one case in point.

In all these analyses, there seem to be a set of 'uniquely human' skills, which, at least in the next few years, will continue to be highly valued while also being less susceptible to automation. Like the workers who will undertake the routine and repetitive tasks, these professional workers may often be doing what is beyond the capabilities of machines or will complement machine activities (Spreitzer et al., 2017). However, unlike the workers who will undertake the routine and repetitive tasks, these professionals' work and capabilities will be highly valued and will be associated with premium wages and working conditions, and a high level of agency in shaping their work and careers (Spreitzer et al., 2017).

\section{$<$ Heading 1> 'Uniquely Human' Skills?}

As technological advances continue, automation will no longer be limited to carrying out tasks that are repetitive and simple. Sophisticated machine-learning systems are starting to be able to accomplish high-level tasks that until recently could be accomplished only by humans, or could not be accomplished at all (Schwab, 2016). Frey and Osborne (2013) suggested that pattern-recognition processes (involving machines learning to classify data in sophisticated ways) would be central to allowing machines to compete with humans in many tasks.

Frey and Osborne (2013) did envision a range of non-routine work activities that were much less likely to be replicated by machines over the next few years, including those involving social and emotional intelligence (e.g., negotiation, care, and persuasion of humans), complex perceptual and manual tasks, and creativity. Autor (2015) and Edmonds and Bradley (2015) also list roles that involve complex problem-solving, interpersonal skills and 'creative intelligence' as being less automatable in the short-to-medium term.

The notion of highly valued human skills is supported by a number of studies of changes in the skill composition of work roles over the last 15 years. MacCrory, Westerman, Alhammadi and Brynjolfsson (2014) examined how roles had shifted in the US between 2006 and 2014. The authors found that, in line with Frey and Osborne (2013), there has been an increase in demand for interpersonal skills and skills required to use and manage machines. In 2015, the World Economic Forum (2016) surveyed employers, and found a predicted sharp increase in demand by the year 2020 for high-level cognitive capabilities, systems skills, and complex problem-solving skills such as mathematics, logical reasoning, visualisation, systems analysis and creativity.

\section{$<$ Heading 1>Creativity: Safe or Not?}

For many scholars, creativity is one of the defining characteristics of what it means to be human, and it tends to be one of the less controversial items in the lists of capabilities suggested to be relatively 'safe' from automation. That said, there continues to be conflict over exactly 
what creativity means, and which types of creativity might be more 'safe'. As Eliza Easton (2018) from the United Kingdom (UK) innovation foundation the National Endowment for Science, Technology and the Arts (Nesta) argues, in many quarters, the term has become so overused as to be meaningless. Easton cites Subway's servers, known as 'sandwich artists', as an example of this.

Recent Nesta studies engage explicitly with the question of whether human creative jobs are machine-proof. In Creativity vs. Robots (Bakhshi et al., 2015), the authors used a methodology similar to that of Frey and Osborne's (2013) study to predict the likelihood of automation of creative job roles. Bakhshi et al. (2015) defined creativity quite broadly, as "the use of imagination or original ideas to create something" (p. 5). Each job role was coded in terms of the probability that it was creative. High-probability creative jobs included creative industries roles that are based on the production of cultural/creative content or services (such as creative artists, filmmakers and architects). The study also included several management occupations, including marketing and sales directors, and advertising account managers. Also included as high-probability creative roles were a range of computer, engineering and science occupations, such as civil engineers, IT specialist managers and chemical scientists. In the US, $21 \%$ of all roles were calculated to be high-probability creative roles, and in the UK, $24 \%$ were highprobability creative roles. In both countries, automation posed minimal risk to $86 \%$ of these workers.

In Creativity and the Future of Skills (Easton \& Djumalieva, 2018), researchers mapped where mention of creativity and creative skills was made in 35 million job advertisements in the UK. The study then used previous research that relied on a combination of machine learning and expert judgement to predict which jobs were most and least likely to grow by 2030 (Bakhshi, Downing, Osborne, \& Schneider, 2017) to show that jobs asking for creativity were far more likely to grow as a percentage of the labour market than other job roles. Other transferable skills, such as detail orientation, basic computer skills and customer service skills were negatively correlated with predicted job growth. By comparison, creative skills paired with digital skills (for non-routine tasks, problem-solving and creating digital content), along with creative skills paired with organisational skills, research and project-management skills, were powerful predictors of job role growth.

\section{$<$ Heading 2>Predicting Future Jobs and Skills: 100 Jobs of the Future}

The Nesta studies are useful in starting to unpack the predicted value of different types of human creativity in the context of a future world of work dominated by technological advancement. A very recent study conducted by Tytler et al. (2019) used a different methodology to explore the kinds of job roles that may exist in 20 years (either newly created or pre-existing). Thematic analysis of the capabilities needed for these job roles reveals more detail about the extent to which, and ways in which, human creativity might be valued in the future.

The researchers recruited experts across technological and research advancements, and domains of application and industries critical to future work. These areas included health, agriculture, engineering and materials science, transport and mobility, computing and AI, commerce, and education. The experts were interviewed, exploring trends in their areas of expertise, potential future job roles, tasks associated with these roles, and the skills and capabilities that young people might develop to prepare for such roles. Transcripts of the interviews were analysed to identify exemplar job roles that captured key elements of what the experts were describing. Overall, 100 job descriptions were constructed that extracted the key 
trends and advancements, roles and tasks, and required capabilities represented in the expert interviews.

The project does not make quantitative predictions about the likelihood or extent of future job roles, and nor does it attempt to be exhaustive in its predictions. Its findings can be useful in describing qualitatively the kinds of human creativity that may be important in the future, along with the relationships between creativity and technology, and with other capabilities. The findings are summarised in Table 15.1.

Table 15.1

100 Jobs of the Future: Jobs Coded by Themes

\begin{tabular}{lc}
\hline Creative job & 82 \\
Primarily creative industries job & 21 \\
Primarily STEM job & 58 \\
Transdisciplinary job & 65 \\
Technology advancement creative job & 15 \\
Technology input creative job & 57 \\
Creative job with technology distribution & 72 \\
Creative job with both technology input and distribution & 35 \\
Creative job with no technology elements & 1 \\
Creative job involving collaboration & 74 \\
Creative job involving social intelligence & 82 \\
Creative job involving organisational skills / project management & 82 \\
\hline
\end{tabular}

Note. $N=100$. Categories overlap so do not add to 100 .

In total, 82 of the job roles were hand-coded as high-probability creative, using the Bakhshi et al. (2015) definition relating to the use of imagination or original ideas to create something, with the addition that the created thing or experience be useful or valued in some way. Of the 82 high-probability creative jobs, nearly one-quarter (21 jobs) fell within the current definition of creative industries roles (Department for Culture Media and Sport, 2015), focusing on production of cultural/creative content or services. Creative industries roles included various kinds of designers (autonomous vehicles, 3D [three-dimensional] printed buildings, gamification, augmented reality experiences), marketers (personalised marketing), content creators (personal brand content) and artists (e.g., swarm artist, who uses swarms of hundreds of drones moving in formation to create art, music or performance-based cultural experiences). A total of 55 of the high-probability creative jobs tended to fall within STEM (science, technology, engineering and mathematics) categories, broadly defined. STEM roles included engineers (bioprinting, mechatronics, additive manufacturing, nanomedical, weather), scientists (terraforming microbiology, biofilm, entomicrobiotech, water management), data or data systems roles (automation anomaly analysis, forensic data analysis, data privacy strategy) and sophisticated kinds of programmers (quantum computers). While some job descriptions in the STEM category called explicitly for 'creativity' or 'creative skills', they were more likely to call for 'problemsolving skills' to create solutions, things or experiences.

Most high-probability creative roles would be categorised primarily into creative industries and STEM roles, but some roles defied disciplinary categorisation. For instance, the 'fusionist' role was described as someone who 
designs approaches to bring together professionals from art, engineering, research, science, and other disciplines to create innovative ideas, experiences, and solutions to complex problems. Fusionists will be employed across many industries, where they will act as bridges between people with specialist disciplinary knowledge. (Tytler et al., 2019, p. 58)

\section{'Trendwatchers' were described as}

those who will know what is likely to happen next, and how to make the most of it. In a future where the pace of change is incredibly rapid, they will be employed by big companies and government agencies to watch the latest developments in science, technology, social issues, and the environment. Trendwatchers will be across the future developments in multiple areas, as new opportunities often occur at the intersection of trends (for example, the development of DNA-based data storage technologies that increase data storage capacity exponentially, at the same time as swarm technologies that allow very big data approaches to city surveillance). (Tytler et al., 2019, p. 60)

Even the high-probability creative roles that could be categorised primarily into creative industries or STEM tended to have strong transdisciplinary elements, with 65 of the 82 jobs requiring capabilities across different disciplinary areas. One example of this is the digital implant designer, who creates 'body hacks' that will be implanted into people's bodies and brains to ensure their health and enhance their lifestyles. Digital implant designers therefore have knowledge of design processes, technical skills relating to implant technologies and digital systems integration, and knowledge of human biology and medical advances.

The high-probability creative roles were also coded in terms of whether they: (a) created technological advances; (b) used technological advances as inputs into creative activities or to distribute creative outputs; or (c) did not rely on technological advances at all-the creative elements of their work were entirely analogue. A total of 15 of the 82 high-probability creative roles created technological advances, through engineering, scientific discovery and/or design processes. Another 57 drew upon technological advances as inputs into creative activities, with a total of 72 relying on technology to distribute creative outputs. Thirty-five roles used technological advances as both inputs and for distribution. Only one creative job role did not rely on technological advances at all: the 'analogue experience guide', who creates experiences for people to

unplug from digital life and reconnect with the natural world, without digital implants or augmented reality. Analogue Experience Guides help people to appreciate a simpler and slower life by experiencing natural environments such as forests or mountainous areas without digital infrastructure or surveillance. (Tytler et al., 2019, p. 91)

Finally, the high-probability creative roles were coded to identify related transferable skills. In line with suggestions by Frey and Osborne (2013) and Bakhshi et al. (2015), social intelligence in different forms was important in the vast majority of high-probability creative jobs in this study. A total of 74 of the creative jobs required collaboration with others, and the 
vast majority of these collaborative relationships involved inputs from people in different roles and from different disciplines. For instance, 'multisensory experience designers'

bring together virtual reality, haptic and biofeedback / biometric technologies to create fully immersive basis of games and leisure activities, marketing campaigns, and education / training. They work in teams with specialists in each of these fields to create experiences that are indistinguishable from the physical world, or alternatively that are incredibly different from the physical world, but are still 'hyperreal'. (Tytler et al., 2019, p. 77)

As in the Bakshi et al., (2015) study, the 74 creative jobs suit project teams which were describe by Caves' (2000) as 'motley crews', with diverse inputs, a combination creative and straightforward tasks and complicated modes of organisation. Thought about like this, it can be seen how creative project-based collaborations (in creative industries, STEM, or transdisciplinary teams) might be resistant to automation. Further, all 82 creative roles (and a total of 97 roles across the whole data set) required social intelligence of some kind, in terms of interacting with other humans beyond any collaborative working arrangements. The social intelligence required differs by role, but involves understanding the needs of others in order to solve problems (nostalgist, cross-cultural capability facilitator), to persuade (negotiator for rights to intellectual property of AI), to sell or market (aesthetician, data commodities broker), to teach (lifelong education adviser) and/or to care and support (100-year counsellor). All 82 creative roles (and a total of 96 across the whole data set) also required organisational and projectmanagement capabilities of some kind, echoing the findings in the Easton and Djumalieva (2018) study.

\section{$<$ Heading $1>$ Creative Skills and Creating the Future}

This chapter has explored some of the deeply divided scholarly opinions about the impact of technological advancements on the future of human work. While some scholars have emphasised the disappearance of job roles under the influence of automation, AI and robotics (some of which has already commenced), others emphasise the creation of new roles that work in concert with new technologies. There is somewhat more agreement about future changes to work arrangements and experiences of work and careers, and also the fact that potential disruptions to work are strongly mediated by the social and economic context. Several authors now suggest that we should not be worried about a relentless 'robot apocalypse', but rather the possibility, under capitalist systems, that the opportunities afforded by technology will be exploited to concentrate wealth, increase income inequality and perpetuate precarious labour conditions.

There do seem to be some capabilities that humans possess that machines do not — at least in the short-to-medium term - and these may be valued in terms of job roles in the future. Creativity is one of these capabilities (although it may be possible in the reasonably short term for machines to be valued for their creativity also, which is probably distinctive from ours). The human creativity in question is a 'generic' type of creativity, that is, the ability for people to create something new that is valued. Creativity of this type can be expressed in the context of creative industries or STEM skills, and often will rely on transdisciplinarity, collaborative processes, social intelligence, and high levels of organisation and project management. This creativity works using technological advances in various ways - through creation of new technologies, technological inputs or technological means of distribution. 
In thinking about the challenges that we face in the coming decades, it may be worthwhile to pivot away from the question of whether jobs may disappear or appear. Rather, it may be useful for us all to think more broadly about how creative humans can work together with machines to promote environmental and economic sustainability, and the well-being of people and communities (Gershenfeld et al., 2017). As Riel Miller (2018), Chief Futurist at UNESCO suggests, rather than trying to forecast or react to a potential future, it is possible to create preferred futures for humans and machines through our considered choices and actions.

\section{$<$ Heading 1 $>$ References}

Autor, D. (2015). Why are there still so many jobs? The history and future of workplace automation. Journal of Economic Perspectives, 29(3), 3-30.

Autor, D., Levy, F., \& Murnane, R. J. (2003). The skill content of recent technological change: An empirical exploration. Quarterly Journal of Economics, 118, 1279-1333.

Bakhshi, H., Downing, J. M., Osborne, M., \& Schneider, P. (2017). The future of skills: Employment in 2030. London, UK: Pearson.

Bakhshi, H., Frey, F., \& Osborne, F. (2015, April). Creativity vs. robots. Retrieved from Nesta website: https://media.nesta.org.uk/documents/creativity_vs._robots_wv.pdf

Borland, J., \& Coelli, M. (2017). Are robots taking our jobs? Australian Economic Review, 50(4), 377-397.

Brynjolfsson, E., \& McAfee, A. (2014). The second machine age: Work, progress, and prosperity in a time of brilliant technologies. New York, NY: Norton.

Caves, R. (2000). Creative industries: Contracts between art and commerce. Cambridge, MA: Harvard University Press.

Cunningham, S., \& Potts, J. (2015). Creative industries and the wider economy. In The Oxford Handbook of Creative Industries (pp. 387-404). Oxford, UK: Oxford University Press.

Department for Culture Media and Sport. (2015). Creative industries economic estimates January 2015 statistical release. Retrieved from https://assets.publishing.service.gov.uk/government/uploads/system/uploads/attachment_ data/file/394668/Creative_Industries_Economic_Estimates_-_January_2015.pdf

Durrant-Whyte, H., McCalman, L., O’Callaghan, S., Reid, A., \& Steinberg, D. (2015). The impact of computerisation and automation on future employment. In Australia's Future Workforce? (pp. 56-64). Melbourne, VIC: Committee for Economic Development of Australia.

Easton, E. (2018). Is creativity the key to the job market of the future? Retrieved from https:/www.britishcouncil.org/anyone-anywhere/explore/digital-creativity/job-marketfuture

Easton, E., \& Djumalieva, J. (2018). Creativity and the future of skills. Retrieved from Nesta website: https://media.nesta.org.uk/documents/Creativity_and_the_Future_of_Skills_v6.pdf

Edmonds, D., \& Bradley, T. (2015). Mechanical boon: Will automation advance Australia? (Office of the Chief Economist Research Paper No. 7/2015). Canberra, ACT: Australian Government Department of Industry, Innovation and Science.

Ford, M. (2015). Rise of the robots: Technology and the threat of a jobless future. New York, NY: Basic Books. 
Frey, C. B., \& Osborne, M. A. (2013). The future of employment: How susceptible are jobs to computerisation? Retrieved from http://sep4u.gr/wpcontent/uploads/The_Future_of_Employment_ox_2013.pdf

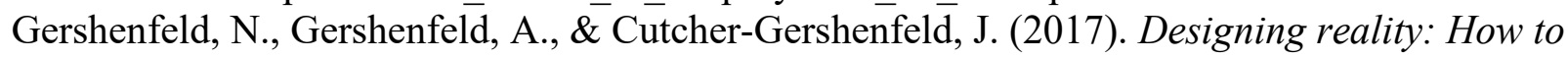
survive and thrive in the third digital revolution. New York, NY: Basic Books.

Hughes, J. (2017). What is the job creation potential of new technologies? In K. LaGrandeur \& J. Hughes (Eds.), Surviving the machine age (pp. 131-145). Champagn, IL: Palgrave Macmillan.

Huws, U. (2014). Labor in the global digital economy: The cybertariat comes of age. New York, NY: NYU Press.

Lent, R. (2018). Future of work in the digital world: Preparing for instability and opportunity. The Career Development Quarterly, 66(3), 205-219.

MacCrory, F., Westerman, G., Alhammadi, Y., \& Brynjolfsson, E. (2014). Racing with and against the machine: Changes in occupational skill composition in an era of rapid technological advance. Proceedings, Thirty Fifth International Conference on Information Systems, New Zealand. Retrieved from https://pdfs.semanticscholar.org/164e/93f0d99852a2b8474c9c0c902eb00807a379.pdf

Manyika, J., Lund, S., Chui, M., Bughin, J., Woetzel, J., Batra, P., ... \& Sanghvi, S. (2017). Jobs lost, jobs gained: Workforce transitions in a time of automation. San Francisco, CA: McKinsey Global Institute.

Miller, R. (2018). Transforming the future: Anticipation in the 21st century. Paris, France: UNESCO Publishing.

Mishel, L., \& Bivens, J. (2017). The zombie robot argument lurches on: There is no evidence that automation leads to joblessness or inequality. Retrieved from Economic Policy Institute website: https://www.epi.org/publication/the-zombie-robot-argument-lurcheson-there-is-no-evidence-that-automation-leads-to-joblessness-or-inequality/

Neufeind, M., O'Reilly, J., \& Ranft, F. (2018). Work in the digital age: Challenges of the fourth industrial revolution. London, UK: Rowan \& Littlefield.

Schwab, K. (2016). The fourth industrial revolution. Geneva, Switzerland: World Economic Forum.

Spencer, D. A. (2018). Fear and hope in an age of mass automation: Debating the future of work. New Technology Work and Employment, 33(1), 1-12.

Spreitzer, G. M., Cameron, L., \& Garrett, L. (2017). Alternative work arrangements: Two images of the new world of work. Annual Review of Organizational Psychology and Organizational Behavior, 4(1), 473-499.

Standing, G. (2011). The precariat: The new dangerous class. London, UK: Bloomsbury Academic.

Susskind, R., \& Susskind, D. (2016). The future of the professions: How technology will transform the work of human experts. Oxford, UK: Oxford University Press.

Tytler, R., Bridgstock, R. S., White, P., Mather, D., McCandless, T., \& Grant-Iramu, M. (2019). 100 jobs of the future. Retrieved from https://100jobsofthefuture.com/report/

World Economic Forum. (2016). The future of jobs: Employment, skills and workforce strategy for the fourth industrial revolution (Global Challenge Insight Report). Retrieved from http://www3.weforum.org/docs/WEF_Future_of_Jobs.pdf

World Economic Forum. (2018). The future of jobs report 2018. Retrieved from http://reports.weforum.org/future-of-jobs-2018/ 